\title{
Design with White Light-Emitting Diodes for an Automotive Low-Beam Projector Headlamp
}

\author{
Yueh-Ying Chiang, Sheng-Jye Hwang, ${ }^{1 *}$ Huei-Huang Lee, \\ Deng-Yuan Hwang, ${ }^{2}$ and Chien-Chih Liao \\ Department of Engineering Science, National Cheng Kung University, \\ No. 1, Daxue Rd., East Dist., Tainan City 701, Taiwan \\ ${ }^{1}$ Department of Mechanical Engineering, National Cheng Kung University, \\ No. 1, Daxue Rd., East Dist., Tainan City 701, Taiwan \\ ${ }^{2}$ Department of Sport, Health and Leisure, Chung Hwa University of Medical Technology, \\ No. 89, Wunhua 1st St., Rende Dist., Tainan City 717, Taiwan
}

(Received December 28, 2015; accepted May 20, 2016)

Keywords: LED headlamp, projection headlamp, ECE R112

A numerical model was developed to design a new automotive low-beam light-emitting diode (LED) headlamp. First, an existing automotive low-beam projector LED headlamp model with ray-tracing software (TracePro) was simulated. The existing commercially available automobile LED headlamp is a combination of one multi-reflector and two projection systems serving as a lowbeam LED headlamp module. The simulated results were compared with the headlamp regulations to prove that the headlamp numerical model and boundary conditions were correct and similar to reality. Finally, the original design was modified into only two projection systems using singlelight-output 515 lumen LEDs. Each projection system was improved to make the headlamp light distributions conform to the ECE R112 regulation. It was concluded that using a high power LED could decrease the number of optical systems required for an automotive headlamp.

\section{Introduction}

The evolution of automotive headlamp design has accompanied the development of light sources. Halogen bulbs have been used as light sources in automotive headlamps since 1970. White light-emitting diodes (LEDs) were invented in 1996, and the LED became a new type of light source, thus initiating solid state lighting. As a new type of light source, LEDs became available for application as automotive headlamps along with the rapid progress in luminous efficiency and heat dissipation technology characteristic of white LEDs. Although the price of LEDs is high, there are many advantages, such as longer lifetime, lower power consumption, short response time, and no mercury pollution, ${ }^{(1)}$ as compared with conventional light sources. Since the luminescence characteristics of LEDs are different from those of conventional light sources, optical systems utilizing LEDs need to be redesigned. As a result, in this research, computer-aided engineering $(\mathrm{CAE})$ was used to simulate the optical performance of an LED headlamp required to conform to the ECE R112 standards. To better understand the performance and applications of the software, a commercially available automobile LED headlamp was studied first. This headlamp

*Corresponding author: e-mail: jimppl@mail.ncku.edu.tw 
was a combination of one multi-reflector and two projection systems serving as a low-beam LED headlamp. The optical system of the LED headlamp was simulated, and the results were compared with existing headlamp regulations to prove that the simulation was representative of reality. Then, the original design was modified so as to contain only two projection systems and LEDs with a single light output of 515 lumens. Each subsequent projection system was improved to make the light distributions of the headlamp conform to ECE R112.

\section{Literature Review}

Inaba et al. (2) succeeded in developing the world's first headlamp incorporating white LEDs installed in the LEXUS LS600H. As shown in Fig. 1, the optical system of this low-beam module consists of three projector units capable of swiveling left and right as an AFS system and one parabola unit. Passive heat sinks are located on the die cast supporting bracket.

In the concept of the multiple lighting unit system, beam distribution is achieved by accurate superimposition of the beam patterns produced by the various contributors. To create the required beam distribution, the axes of the four units must be accurately aligned. As shown in Fig. 2, misaligned optical axes can occur. As shown in Fig. 3(a), two elements influence the accurate alignment of the optical axes.

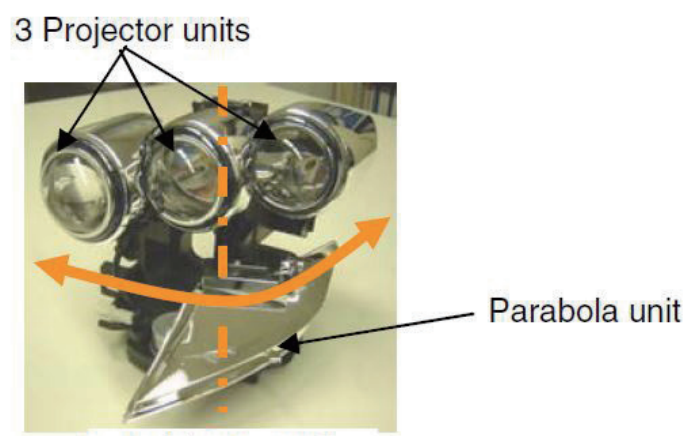

Fig. 1. (Color online) Low-beam unit.(2)

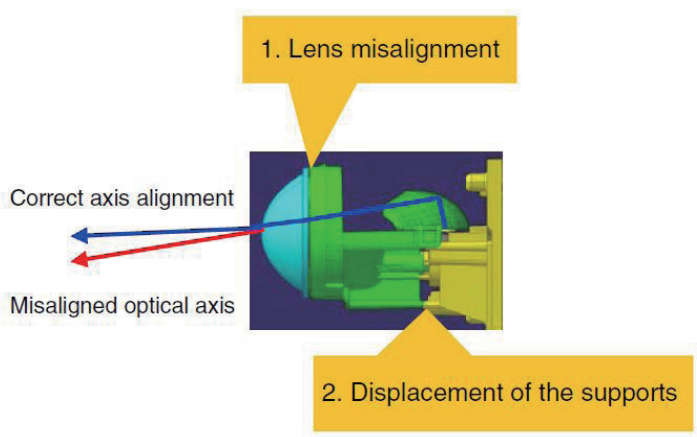

(a)

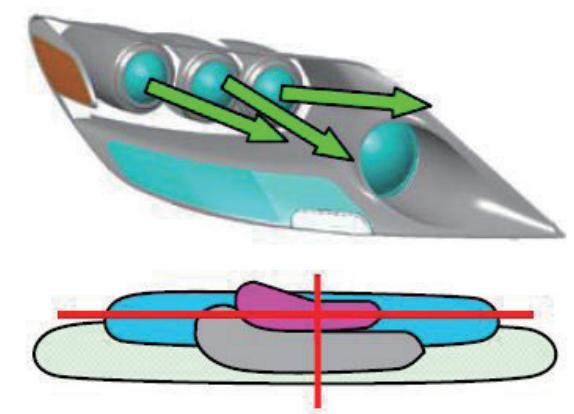

Fig. 2. (Color online) Misaligned optical axes. ${ }^{(2)}$

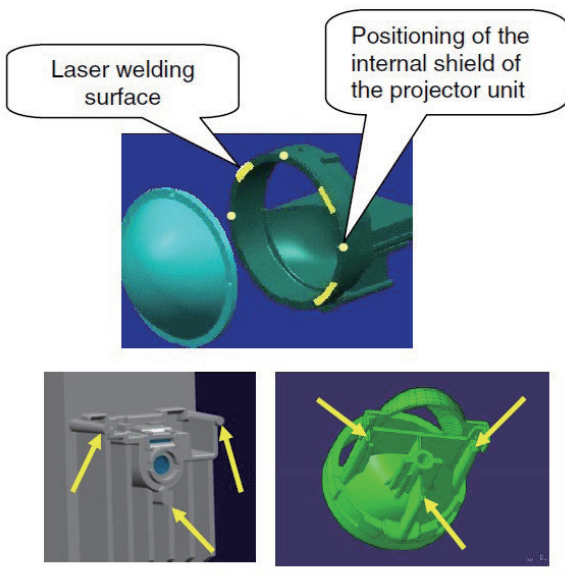

(b)

Fig. 3. (Color online) (a) Factors contributing to optical axis misalignment. (b) The method for solving misaligned optical axes. ${ }^{(2)}$ 
As shown in Fig. 3(b), to increase the accuracy of the positions of the lens and the internal projector unit shield, a positioning mechanism with highly accurate pins and holes is provided. The assembly is then completed by laser welding and by using only three welding areas so that flatness after welding is enhanced.

As shown in Fig. 3(b), to increase the accuracy of the optical unit support system, a 3-point arrangement is provided for the assembly of the optical units and the brackets used to fix the optical axis position.

\section{Research Procedures}

\subsection{Reverse engineering of LED headlamp}

First, a commercially available LED headlamp as shown in Fig. 4 was simulated. This headlamp was a combination of three optical systems; one multi-reflector, and two projection systems. The optical projection system is a combination of a light source, a reflector, a shade, and a lens, ${ }^{(3)}$ as shown in Fig. 5.

Assuming that the original LEDs were from a Lambertian source, the light output for each was 350 lumens; the spatial distribution is shown in Fig. 6. The effect on the surface absorption of the reflector and shade was ignored, and surface reflectivity was set at $100 \%$. The lens material was assumed to be glass (Schott BK7), and the relationship between the wavelength and the indices of refraction is shown in Fig. 7.

\subsection{New LED headlamp design}

In the new LED headlamp design, high power LEDs were used, and this LED model was built based on its spectral power distribution and spatial distribution datasheet in TracePro, as shown in Figs. 8 and 9. The LED geometry is shown in Fig. 10, the details of the LED information are shown in Table 1. The light output in TracePro was set at 500 lumens. The new LED headlamp design is a combination of two projection systems, as shown in Fig. 11. The design is possible because the LEDs used in the new design are much brighter and have higher efficiency than the ones used previously.

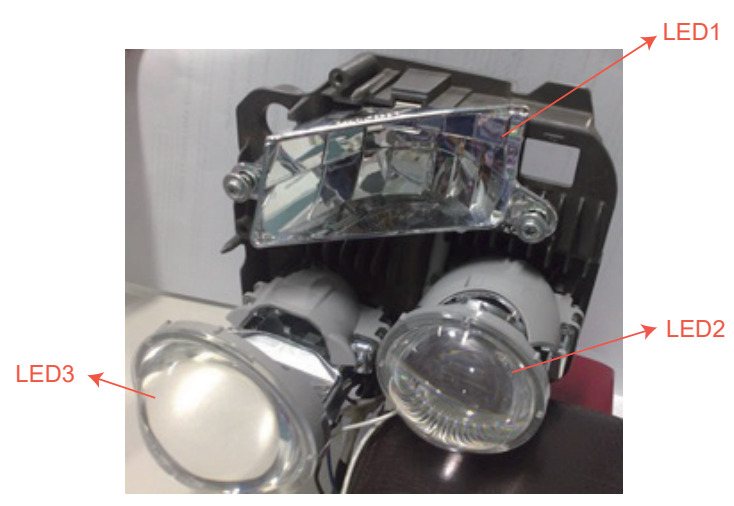

Fig. 4. (Color online) Original LED headlamp.

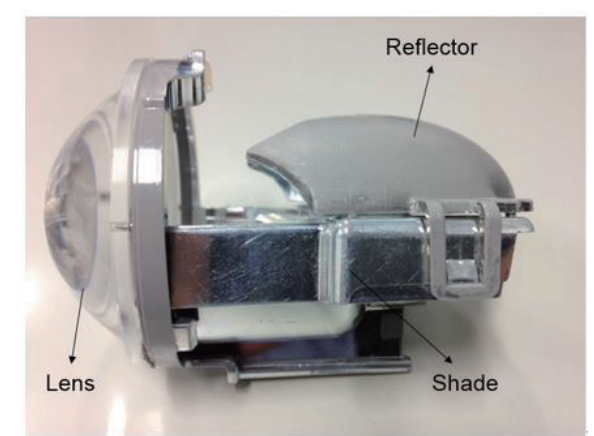

Fig. 5. (Color online) Optical projection system. 


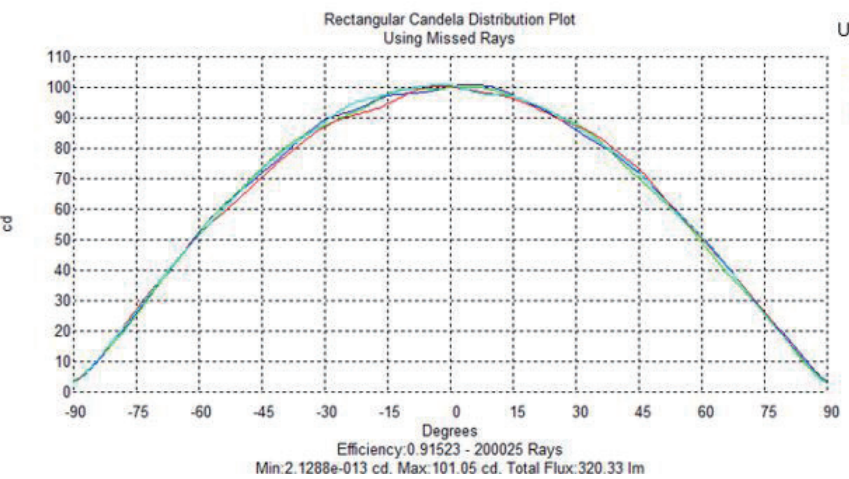

Fig. 6. (Color online) Spatial distribution of the Lambertian source.

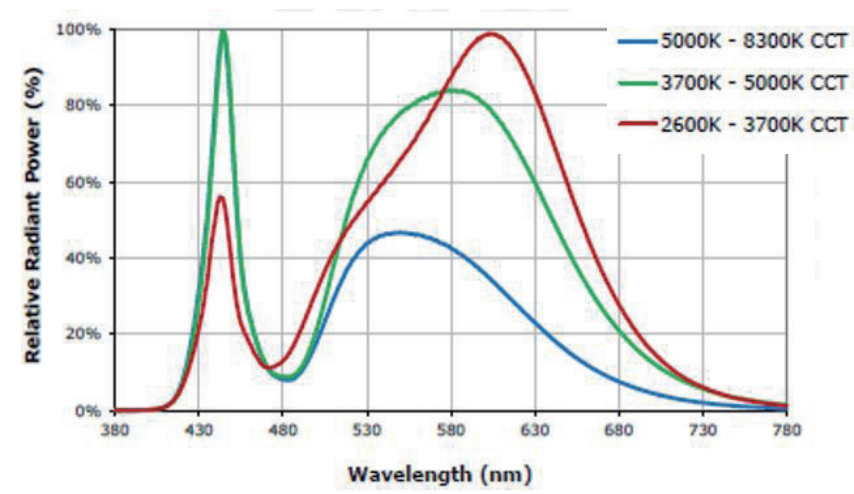

Fig. 8. (Color online) Spectral power distribution of LEDs. ${ }^{(3)}$

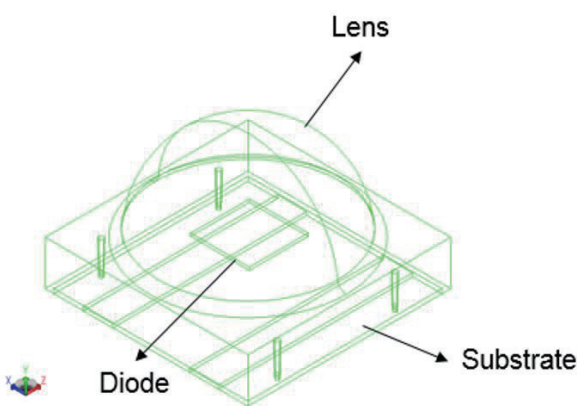

Fig. 10. (Color online) Spatial distribution of LED. ${ }^{(3)}$

Table 1

Information about the LED. ${ }^{(3)}$

\begin{tabular}{lc}
\hline Maximum drive current & $1.5 \mathrm{~A}$ \\
Maximum power & $4.9 \mathrm{~W}$ \\
Light output & $515 \mathrm{~lm} @ 4.7 \mathrm{~W}\left(85^{\circ} \mathrm{C}\right)$ \\
Maximum junction temperature & $150{ }^{\circ} \mathrm{C}$ \\
\hline
\end{tabular}

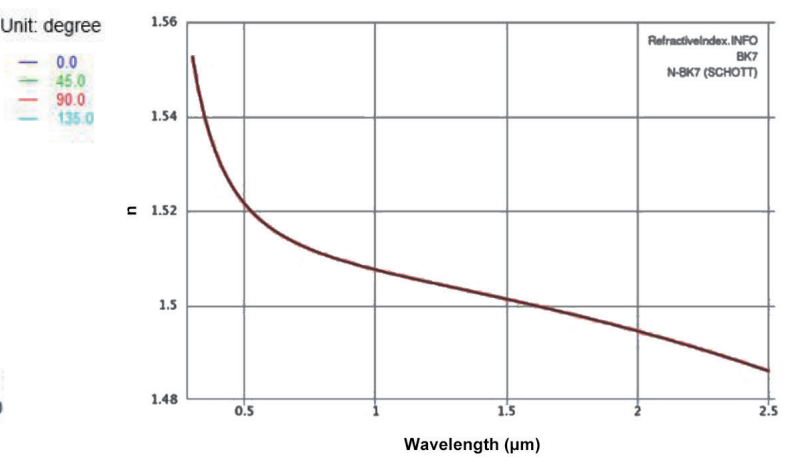

Fig. 7. (Color online) BK7 indices of refraction.

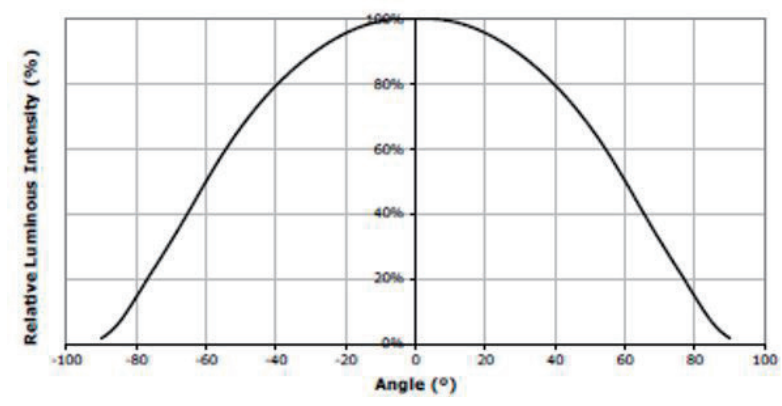

Fig. 9. (Color online) Spatial distribution of LEDs. ${ }^{(3)}$

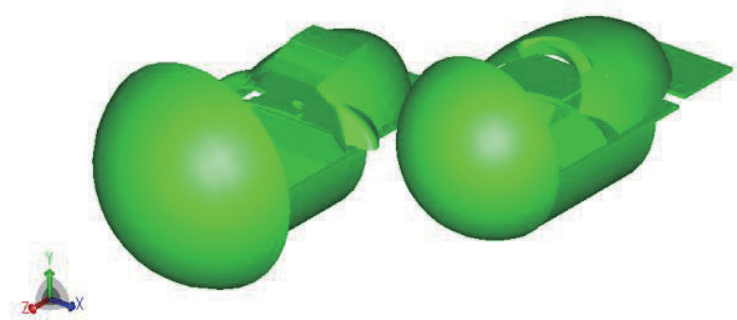

Fig. 11. (Color online) New low-beam LED headlamp design. 


\section{Results and Discussion}

\subsection{Light shape in simulation}

Figure 12(a) illustrates the shape of the light in the experiment and the simulation for the original LED headlamp. A comparison between the shape of the light in the experiment and in the simulation indicated that they were similar and that the simulation was realistic. Figure 12(b) shows the shape of the light of the new LED headlamp design. In a comparison with the results of the original LED headlamp, it can be seen that the shapes of the lights are similar.

\subsection{Modified model of optical systems}

For the new headlamp design, the degree of the surface behind the shutter was changed to $3^{\circ}$ of LED 3 to concentrate the light under the cut-off line, as shown in Fig. 13. The shape of the light above the cut-off line was made smoother by modifying the structure of A and B on the shutter, as

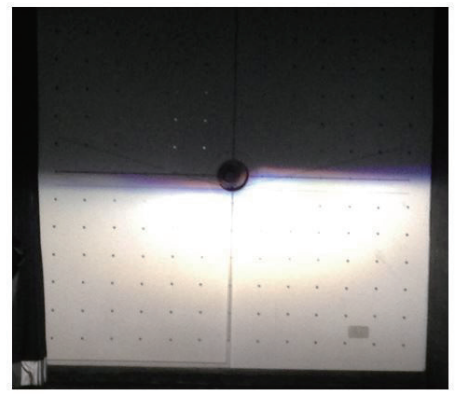

Experiment

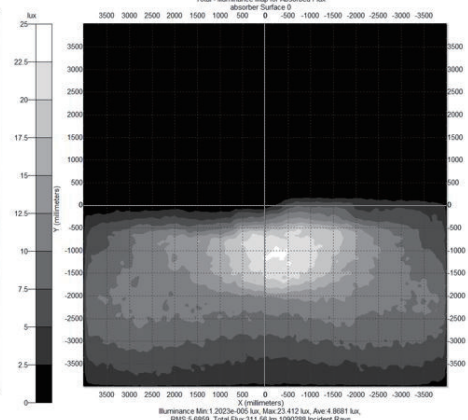

Simulation

(a)

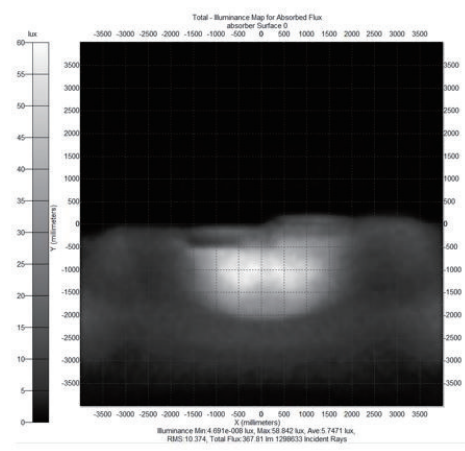

(b)

Fig. 12. (Color online) (a) The shape of the light in the original LED headlamp experiment and simulation. (b) The shape of the light in the new LED headlamp design for the simulation.
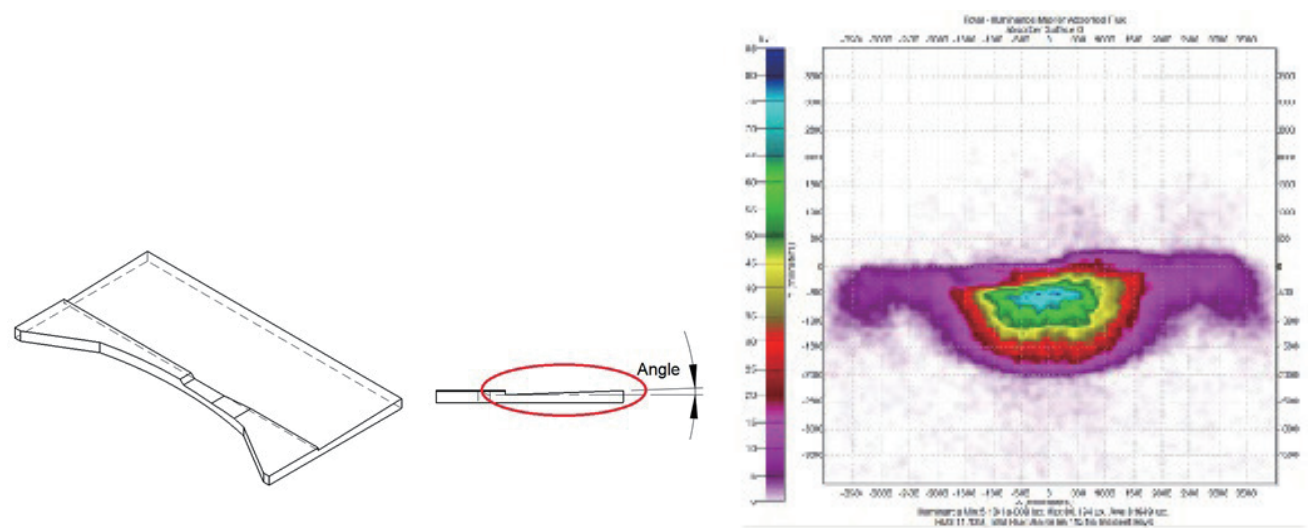

Fig. 13. (Color online) Light shape of LED 3 after modification of the front parts of the shutter. 
shown in Fig. 14. The height of the parts $4 \mathrm{~mm}$ from the centerline of structure A was modified to 1.15 $\mathrm{mm}$, and a $3.20 \times 2.15 \mathrm{~mm}^{2}$ absorbing surface was added on the shutter to create a smooth light shape on the left of cut-off line on the screen. The prominent parts of structure B were removed, and the parts $4.9 \mathrm{~mm}$ from the centerline were lowered to $0.2 \mathrm{~mm}$ to create a smoothing light shape on the right of screen, as shown in Figs. 15(a) and 15(b).

The degree of the cant was modified to $40^{\circ}$, and the width of the structure below LED 2 was modified to $12.8 \mathrm{~mm}$, and the new light shape is shown in Fig. 16. A region of the dark side of the

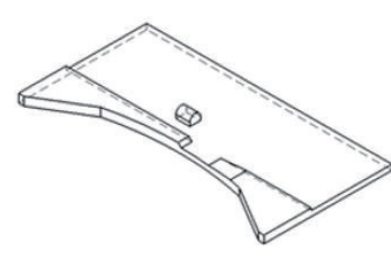

Unit: $\mathrm{mm}$
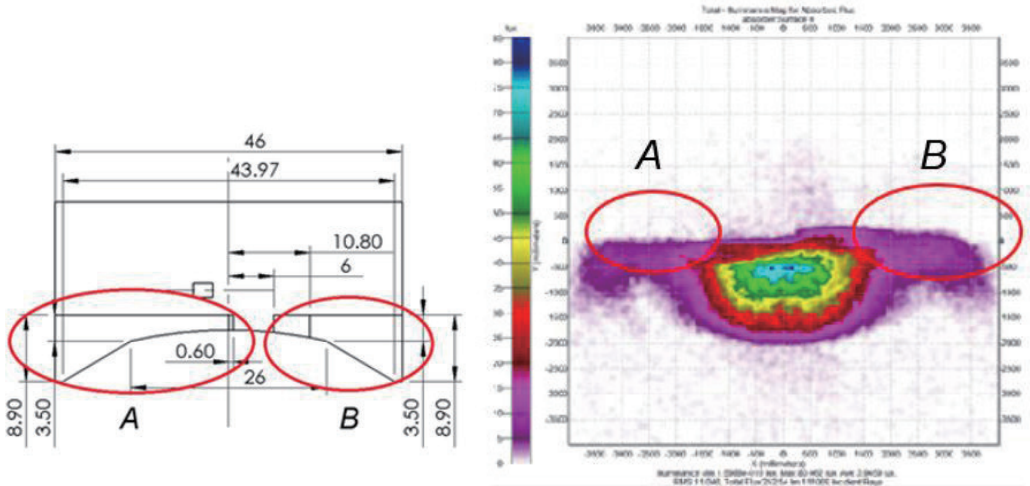

Fig. 14. (Color online) Light shape of LED 3 after modification of the rear parts of the shutter.

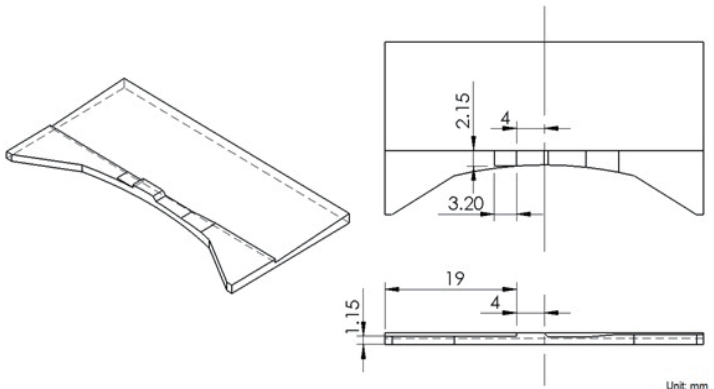

(a)

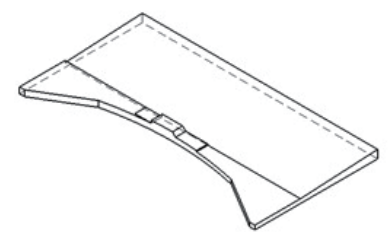

(b)

Fig. 15 (a) The final design of part A of the shutter of LED 3. (b) The final design of part B of the shutter of LED 3.
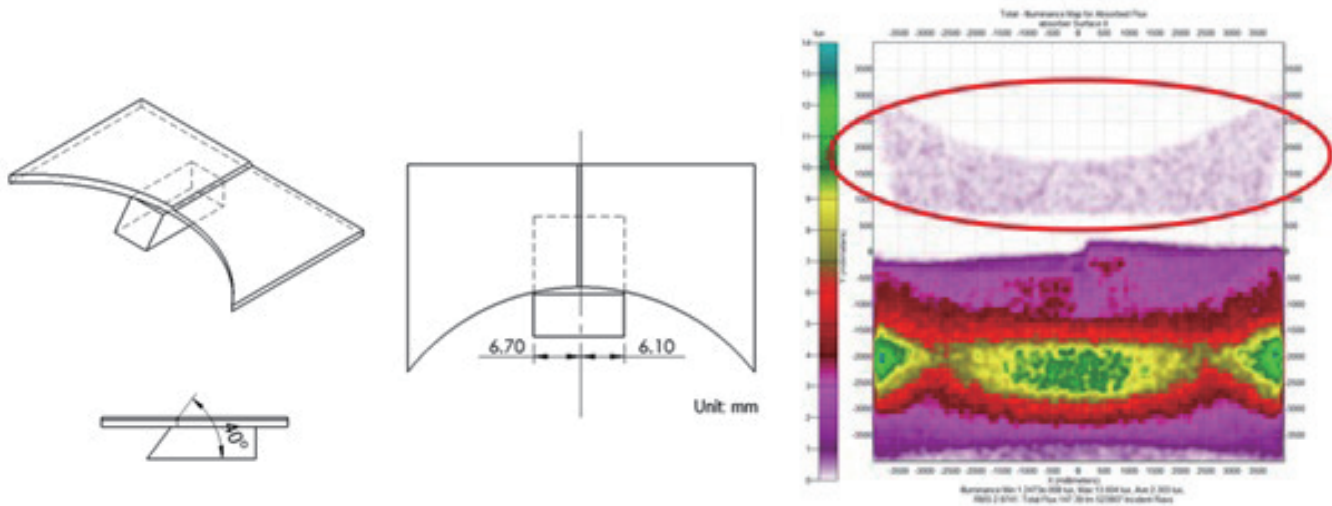

Fig. 16. (Color online) LED 2 light shape after modification of the lower parts of the shutter. 
screen having uniform light exposure with 0.3 Lux allowed the illuminance of check points $1+2+3$ and $4+5+6$ to meet the standards set in the regulation.

To remove the light above the cut-off line on the screen, the degree of the cant was modified to $9^{\circ}$, around the shutter on the LED 3. The axis of the LED 3 optical system was rotated $0.236^{\circ}$ horizontally downward and turned right vertically $0.229^{\circ}$ to meet the regulations for zone III, as shown in Fig. 17.

After the modification of the LED 2 and LED 3 optical systems, the new results for the light shape simulation were as shown in Fig. 18. The illumination for each check point on the screen was compared with the ECE R112 standard as listed in Table 2, which shows that all meet the standard.

\subsection{Check points for the ECE R112 standard}

According to the ECE R112 standard, engineers have to check the illumination at several points and areas to see whether a lamp meets the standards. As shown in Table 2, although some check

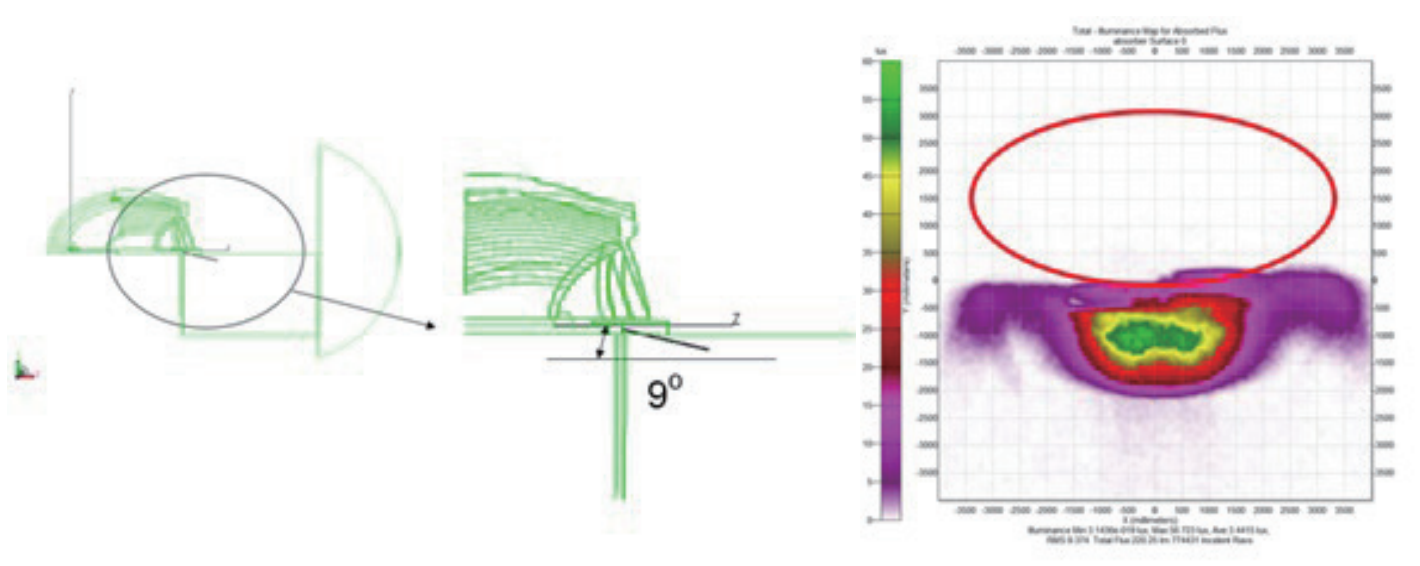

Fig. 17. (Color online) LED 3 light shape after modification.

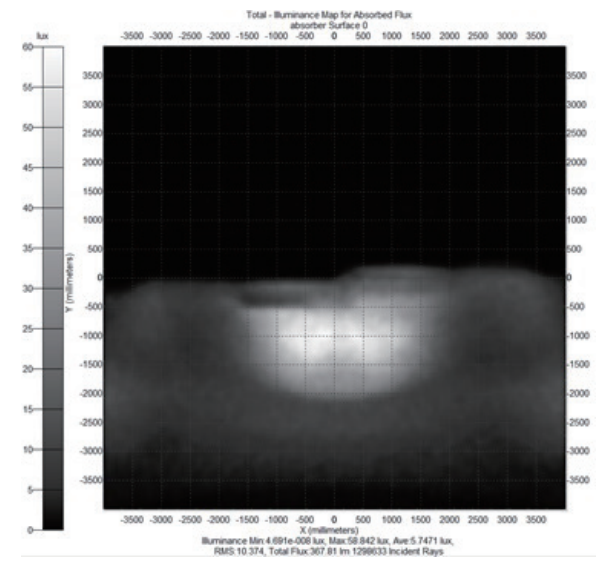

Fig. 18. (Color online) LED headlamp light shape with the new design. 
Table 2

Simulation results of illumination values for each check point.

\begin{tabular}{lccc}
\hline Check Point & $\begin{array}{c}\text { Standard } \\
\text { (Unit: lux) }\end{array}$ & $\begin{array}{c}\text { Simulation results of } \\
\text { original model } \\
\text { (Unit: lux) }\end{array}$ & $\begin{array}{c}\text { Simulation results of } \\
\text { new design model } \\
\text { (Unit: lux) }\end{array}$ \\
\hline B50L & $\leq 0.4$ & 0.6 & 0.1 \\
75R & $\geq 12$ & 11.1 & 20.1 \\
75L & $\leq 12$ & 3.5 & 9.7 \\
50R & $\geq 12$ & 14.0 & 30.2 \\
50L & $\leq 15$ & 5.2 & 5.4 \\
$50 \mathrm{~V}$ & $\geq 6$ & 10.0 & 20.3 \\
25R & $\geq 2$ & 4.6 & 5.6 \\
25L & $\geq 2$ & 4.7 & 3.8 \\
Any point in zone III & $\leq 0.7$ & $3.2 \%$ (fail rate) & $0.0 \%$ (fail rate) \\
Any point in zone IV & $\geq 3$ & $0.0 \%$ (fail rate) & $0.0 \%$ (fail rate) \\
Any point in zone I & $\leq 2 \mathrm{E}^{*}$ & $0.0 \%$ (fail rate) & $0.0 \%$ (fail rate) \\
$1+2+3$ & $\geq 0.3$ & 0.4 & 0.4 \\
$4+5+6$ & $\geq 0.6$ & 0.2 & 0.7 \\
7 & $0.7 \geq 7 \geq 0.1$ & 1.4 & 0.2 \\
8 & $0.7 \geq 8 \geq 0.2$ & 1.2 & 0.5 \\
\hline
\end{tabular}

$\mathrm{E}^{*}$ is the real measured value of illuminance on point $50 \mathrm{R}$ or $50 \mathrm{~L}$.

points failed to meet the standards, the trend of the performance for the original LED headlamp was close to the standard. The failures could be due to inaccuracy in the lamp geometry since the geometry was obtained via reverse engineering. However, the results prove that the setup with TracePro used to simulate the performance of light distribution for LED headlamps was appropriate. Figure 12(b) and Table 2 provide the simulation results for the newly designed LED headlamp. The results indicate that the performance of the new design for the LED headlamp conforms to the ECE R112 standard.

\section{Conclusions}

This study made use of TracePro to simulate the performance in terms of light distributions for an LED automotive headlamp design, and ensured that the light shape meets the regulation. Details of the conclusions are:

(1) The LED headlamp design was proven to be appropriate via simulation software.

(2) LEDs with higher luminescence efficiency can reduce the number of optical systems in a headlamp.

(3) Using only two projection systems as a LED headlamp is possible if a single LED light output reaches more than 500 lumens.

(4) The volume of the new design was $40 \%$ smaller than the original LED headlamp.

\section{References}

1 B. Kang, K. Park, B. Yong, and H. Kim: SAE International, Paper (2007) No. 2007-01-3516.

2 T. Inaba, S. Watanaba, and Y. Yamada: SAE International, Paper (2008) No. 2008-01-0339.

3 CREE: Cree ${ }^{\circledR}$ XLamp ${ }^{\circledR}$ XP-G2 LEDs, Product Family Data Sheet (CREE, Durham, U.S., 2012). 